\title{
Emergencias oftalmológicas y su manejo por el médico general
}

\section{Ophtalmology emergencies and its management by the general practitioner}

\author{
Guillermo Barriga-Salaverry', Guillermo C. Barriga-Doy²
}

\section{Resumen}

Las emergencias oftalmológicas son situaciones repentinas con riesgo inminente de pérdida de la visión que requieren atención inmediata para evitar secuela permanente. Algunas de ellas deberán ser atendidas en primera instancia por un médico emergencista. El objetivo de esta sección es proporcionar los elementos diagnósticos y terapéuticos para que todo médico pueda realizar la tarea con éxito en esta instancia y derivar la situación en forma segura y oportuna al oftalmólogo.

Palabras clave: Dolor ocular, queratoconjuntivitis, cuerpo extraño, traumatismo ocular.

\section{Abstract}

Ophthalmological emergencies are sudden situations with imminent risk of vision loss that require immediate attention to avoid permanent sequelae. Some of them must be attended in the first instance by a emergency doctor. The objective of this section is to provide the diagnostic and therapeutic elements so that every physician can perform the task successfully in this instance and derive the situation in a safe and timely manner to the ophthalmologist.

Keywords: Eye pain, keratoconjunctivitis, strange body, ocular traumatism.

Introducción

La emergencia oftalmológica (EO) se define por la situación de salud ocular que se presenta repentinamente y que, llevando una alta probabilidad de riesgo de pérdida de la visión, pal requiere atención y tratamiento inmediato. Este concepto debe diferenciarse de la urgencia oftalmológica que se refiere a la situación de salud ocular que también se presenta repentinamente, pero sin riesgo de pérdida de la visión y que puede requerir de una asistencia oftalmológica dentro de un período de tiempo razonable de dos o tres horas ${ }^{(1)}$.

Las emergencias oculares más comunes que se presentan son los traumas oculares, el desprendimiento de retina y la oclusión de la arteria central de la retina. En Estados Unidos de Norteamérica se reportan 2 millones de traumas al año ${ }^{(3)}$. En el 2011 se reportaron 8361 emergencias oftalmológicas (EOs) en 5 años en una población egipcia ${ }^{(2)} \mathrm{El}$ $69 \%$ ocurrieron en varones y el tipo de EO más frecuente fue el trauma ocular con el $33.46 \%$, ocupando el primer lugar en ambos sexos, seguido de queratitis infecciosa y laceración palpebral para los varones, y de queratitis infecciosa, endoftalmitis y glaucoma para las mujeres ${ }^{(2)}$. Según los grupos de edad, el 24\% de los EOs fueron en el grupo de 6 a 16 años, en los mayores de 45 años, el 26.8\%. En la estratificación por edad y tipo de EO, en los menores de 45 años fue más frecuente el trauma ocular abierto con el $36 \%$ y en los mayores de 44 años la queratitis infecciosa con el $34.4 \%$.

La mayoría de los casos de EOs pueden examinarse con herramientas tan sencillas como una cartilla de Snellen (o su equivalente en aplicaciones de celulares como el Peek Acuity), una linterna de bolsillo y un oftalmoscopio directo, que no debería faltar en el maletín de todo médico.

${ }^{1}$ Médico Oftalmólogo, Maestro en Medicina, Profesor Principal y Director Coordinación Docente Hospital A. Loayza Universidad Peruana Cayetano Heredia, Ex-Jefe del Servicio de Oftalmología del Hospital A. Loayza, Académico de Número Academia Peruana de Cirugía. ${ }^{2}$ Médico Oftalmólogo, Postgrado en retina, Médico asistente de la Clínica de Utrera, España. 
Con la Cartilla de Snellen o la Aplicación Peek Acuity, para teléfonos celulares, se realiza la medición de la agudeza visual en diversas escalas, aportando el dato más importante para definir la severidad de una EO. Su uso requiere un tiempo de aprendizaje muy corto y su acceso es universal y la aplicación Peek Acuity es de distribución gratuita.

La linterna de mano aporta información sobre el segmento anterior del ojo, el estado de la superficie corneal y anexos, así como de los reflejos pupilares, que permiten diferenciar las pérdidas de visión por lesiones maculares, que no los altera, de las lesiones del nervio óptico, que producen un defecto pupilar aferente en la mayoría de las veces, apuntando además a un compromiso concomitante del sistema nervioso central.

Contar con un oftalmoscopio directo debería ser tan básico como contar con un estetoscopio, con la diferencia que éste último podemos reemplazarlo con nuestro oído sobre el tórax del paciente. El oftalmoscopio en cambio no puede ser reemplazado. Su utilidad radica en la información que puede determinar la evaluación del reflejo rojo pupilar para estimar la transparencia de los medios oculares y fundamentalmente para examinar el fondo de ojo, donde se puede diagnosticar el $90 \%$ de las enfermedades sistémicas que tienen manifestaciones en la retina y el $100 \%$ de las que se manifiestan en el nervio óptico.

\section{EMERGENCIAS OFTALMOLÓGICAS}

Los síntomas y signos de alarma de las EOs son fundamentalmente la disminución de la agudeza visual (AV), el dolor y el enrojecimiento ocular ${ }^{(2)}$. Particularmente puede ser importante el hallazgo de una pupila dilatada y fija o la presencia de una evidente lesión corneal asociado a estos síntomas ${ }^{(3)}$

La disminución de la $\mathrm{AV}$ puede reportarse como permanente o esporádica. Medida con una cartilla de Snellen que debería estar disponible en todo consultorio o sala de emergencia. Debe ser preocupante cualquier AV menor de $20 / 40$, teniendo en cuenta que a menor AV al momento del diagnóstico, peor pronóstico funcional y más urgente la necesidad de evacuación a un centro especializado.

El dolor ocular se caracteriza en función de su intensidad y a su irradiación al cráneo. Algunas situaciones como el glaucoma agudo puede producir inclusive shock neurogénico por su severidad ${ }^{(2)}$. Por lo general asociado al dolor se puede registrar fotofobia, blefarospasmo y lagrimeo.

El enrojecimiento ocular se debe evaluar en función a su extensión, focal o difuso, y a su asociación a disminución de la $\mathrm{AV}$ y presencia de dolor. Particular importancia debe recibir la observación de hiperemia periquerática, que consiste en la congestión de los vasos limbares radiales a la córnea que representan inflamación seria del cuerpo ciliar y estructuras vecinas, asociada con frecuencia a la severidad del daño de la EO.

La EOs pueden llegar en primera instancia a consultorios o servicios de emergencias donde no haya un oftalmólogo, pero que por su naturaleza y evolución natural debería ser diagnosticada y tratada por un médico no oftalmólogo; de lo contrario, ocurriría una pérdida de la visión que en algunos casos podría ser irreversible.

Es en ese sentido el objetivo de esta publicación, que pretende proporcionar un instructivo diagnóstico y terapéutico de dos de las principales emergencias oftalmológicas, donde el médico a cargo, sin ser especialista, debe diagnosticar y tratar el problema de salud ocular que ha recibido. Se trata del Glaucoma agudo congestivo y la Queratoconjuntivitis química.

\section{GLAUCOMAAGUDO CONGESTIVO}

Es una situación crítica de emergencia ocular en donde, por razones de origen anatómico, ocurre una interrupción repentina del drenaje del humor acuoso de uno de los ojos, ocasionando una elevación súbita de la presión ocular que deviene en un síndrome caracterizado por dolor y enrojecimiento oculares asociado a pérdida de la visión ${ }^{(6)}$. El examen revelará hiperemia periquerática, opalescencia de la córnea y una pupila midriática, de 4 a $5 \mathrm{~mm}$ de diámetro, sin respuesta al estímulo luminoso. La presión sobre el globo ocular afectado no permite deprimirlo, apreciándose duro y doloroso por la maniobra. Conforme aumenta la presión ocular, el dolor se incrementa, pudiendo desencadenar en pérdida transitoria del conocimiento y agravar la pérdida de la visión por obstrucción de la arteria central de la retina.

Estos hechos determinan la necesidad de tratamiento inmediato e impostergable de parte del médico que atiende la emergencia, cuyo objetivo es bajar la presión intraocular, que se consigue con la administración de $1 \mathrm{cc} / \mathrm{kg}$ de peso de glicerina al $20 \%$ por vía oral, diluido en una bebida que enmascare su sabor desagradable, produciendo una diuresis osmótica y la consecuente deshidratación del cuerpo vítreo, que ocurre aproximadamente 30 a 45 minutos después de ingerido el brebaje.

Calmado el dolor, puede deducirse la normalización de la presión intraocular y preparar la evacuación para la atención del especialista, que en la mayoría de los casos consistirá en una iridotomía realizada con láser, seguida de una cirugía filtrante para glaucoma ${ }^{(7)}$.

\section{QUERATOCONJUNTIVITIS QUIIMICA}

Es la inflamación tóxica de la córnea y la conjuntiva de un ojo producida por el contacto con una sustancia química de propiedades corrosivas que desnaturaliza las proteínas de los tejidos afectados. Sobre la naturaleza de la sustancia química implicada es importante conocer su $\mathrm{pH}$ porque se sabe que las sustancias alcalinas (amónico, lejía, cal) son más dañinas que las ácidas a concentraciones parecidas y el pronóstico de los daños que ocurrieran es más pobre cuando el agente agresor fue unálcali ${ }^{(4)}$.

Sin embargo, el manejo terapéutico de cualquier sustancia tóxica se inicia igual en todos los casos, buscando 


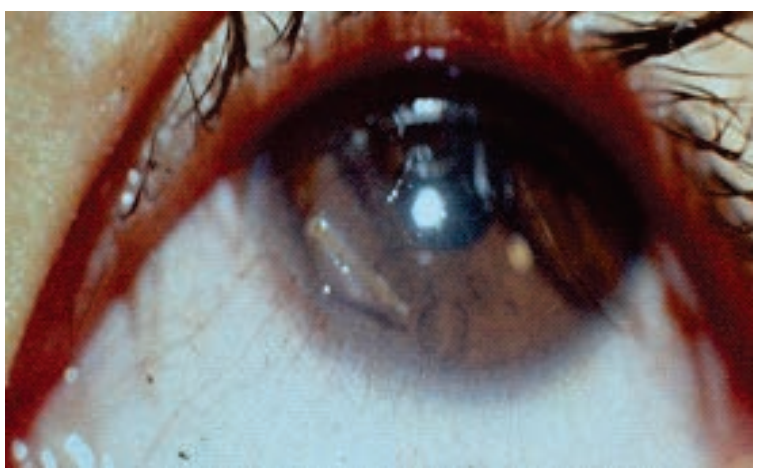

Cuerpo extraño vegetal superficial.

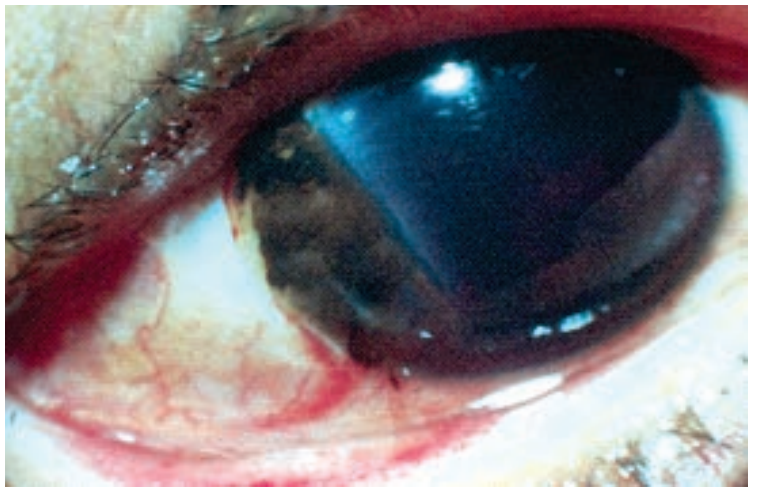

Herida corneal con hernia de iris

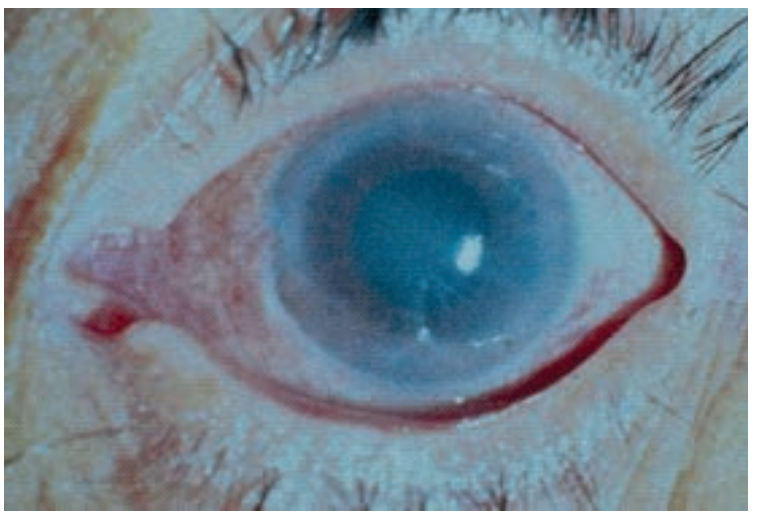

Glaucoma Agudo congestivo.

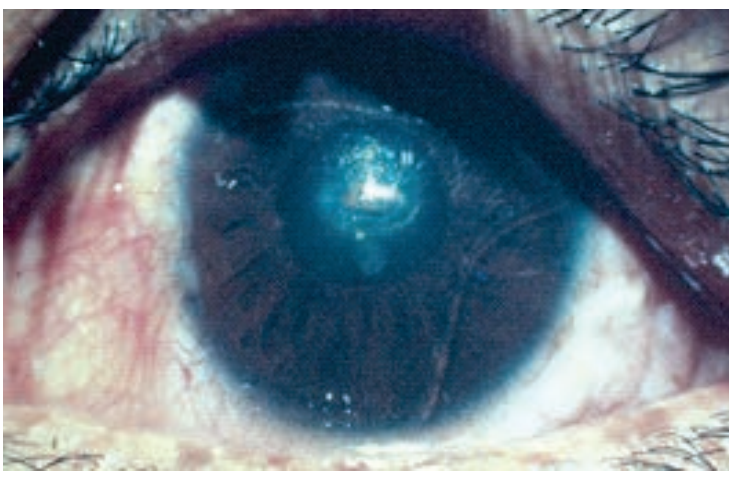

Cuerpo extraño metálico profundo.

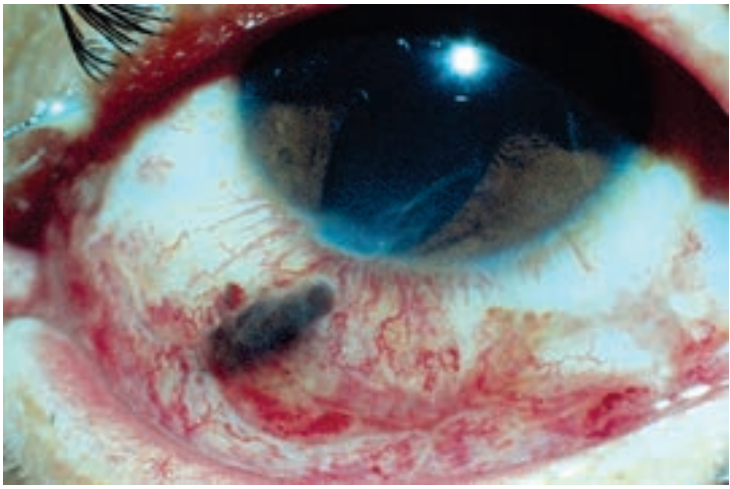

Herida escleral, pupila deformada y salida de iris en esclera.

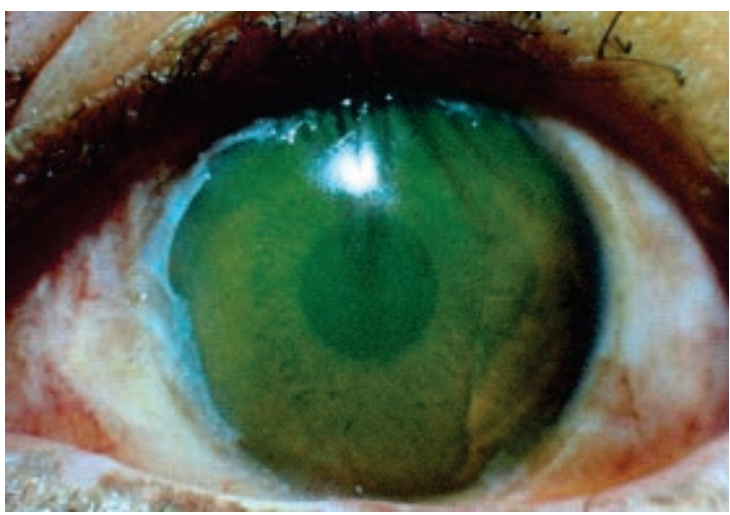

Quemadura química por ácido.

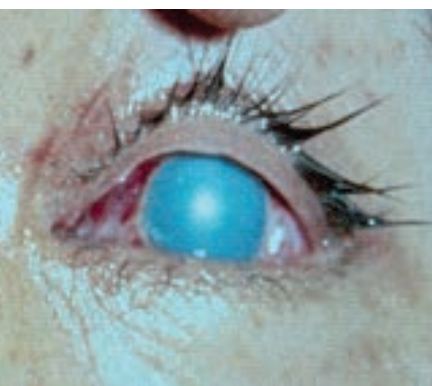

Grave secuela de quemadura corneal por álcali que no fue tratada. 
diluir su concentración y aproximarse al pH neutro. Esto se consigue en el mejor de los casos con una solución isotónica de cloruro de sodio estéril, que no alterará el equilibrio osmótico de los tejidos afectados y se irriga de manera profusa y contínua sobre la superficie del ojo afectado. Se sabe que con el uso de agua potable o agua destilada se puede conseguir la dilución más rápida de cualquier solución, sin embargo, el agua así usada, puede alterar la osmolaridad de la mucosa conjuntival y la córnea, desencadenando un edema osmótico que puede enmascarar la inflamación que acompaña a las quemaduras por ácidos o álcalis.

Paralelamente con la medida descrita, deberá administrarse antinflamatorios no esteroideos y analgésicos por vía oral, además de cubrir con antibióticos tópicos en forma de ungüento las quemaduras producidas, evitando en lo posible, apósitos sobre el ojo. Oportunamente y cuando ya esté estabilizado el paciente, deberá referirse al especialista correspondiente para evaluar la reparación, probablemente quirúrgica, de los daños y secuelas.

Finalmente, hay un grupo de EOs por las que el médico no especialista deberá hacer un diagnóstico aproximado, pero no deberá hacer tratamiento alguno por la necesidad de contar con un oftalmólogo y los equipos e instrumental apropiados que requiere el manejo de estas condiciones ${ }^{(4)}$. En consecuencia, todas ellas deberán ser referidas al especialista de inmediato. Se trata de las siguientes situaciones:

Cuerpo extraño, de origen orgánico en la córnea: que requiere la remoción inmediata bajo microscopio para evitar su profundización y reducir el inóculo de gérmenes que podría alcanzar el interior del ojo, sobre todo hongos que pueden hacer mucho daño al ojo afectado ${ }^{(2)}$.

Obstrucción de arteria central de la retina: asociada a una pérdida aguda de la visión, con defecto pupilar aferente y un fondo de ojo característico en que se observa "la mancha rojo cereza" en la mácula que puede encontrarse usando el oftalmoscopio directo. Muchas veces se encontrará factores de riesgo asociados como hipertensión arterial, dislipidemias o accidentes vasculares previos como el infarto agudo de miocardio y/o accidentes cerebro vasculares. Estos últimos con muchas probabilidades de recurrir en los próximos 5 años después del evento ocular. Existen reportes de recuperación de estos casos con maniobras oftalmológicas extremas si el paciente es evacuado a un oftalmólogo dentro de las 2 horas de ocurrido el evento.

Desprendimiento de Retina: igualmente asociado a una pérdida repentina de parte o todo el campo visual del ojo afectado. Cuando es parcial, el paciente aqueja ver una sombra como el de una cortina que cae o se levanta de su campo visual. En los casos totales habrá amaurosis franca, hipotensión ocular notándose el ojo muy blando y eventualmente leucocoria. Por lo general se recogerá información de un trauma ocular, craneal o facial previo a la pérdida de la visión. Un examen con linterna confirmará la leucocoria y eventualmente un defecto pupilar aferente si el desprendimiento de retina ha sido total. El examen con el oftalmoscopio directo revelará el desprendimiento correspondiente si ha alcanzado el polo posterior. $\mathrm{Su}$ derivación al especialista debe ser inmediata para el tratamiento quirúrgico correspondiente. El concepto a recordar para estos casos es que mientras más tiempo se difiera el tratamiento quirúrgico, menos probabilidades de recuperación visual habrá ${ }^{(t)}$.

Laceración corneal: es una herida perforante de la córnea, a través de la cual se pierde humor acuoso y, por lo tanto, existe el riesgo potencial de aplanamiento de la cámara anterior e incarceración del iris en el defecto corneal ${ }^{(2)}$. La reparación de la herida no admite retraso y solo puede hacerla el oftalmólogo con el equipo apropiado, en consecuencia, la evacuación de estos casos debe ser inmediata.

Traumatismo ocular con pérdida de la visión: la mayoría de estos casos corresponde a pacientes politraumatizados en accidentes de tránsito, en quienes la prioridad siempre será estabilizarlos hemodinámicamente, tomando las primeras horas del manejo del equipo de guardia ${ }^{(5)}$. Cuando se sospeche que se trata de una herida ocular abierta o con exposición de contenido intraocular no debe insistirse con el examen porque puede empeorar la situación anatómica del ojo afectado. En estos casos corresponde cubrir sin presión con apósitos estériles el o los ojos traumatizados y cursar una interconsulta urgente a oftalmología ${ }^{(8)}$.

Referencias bibliográficas

1. Duke Elder. System of Opthamology. St. Louis U.S.A. 1965;8.

2. Haro DE. Emergencias Oftalmológicas. Principios y Terapéutica Quirúrgica, Editor E. Barboza. Imp. Didí de Arteta. Lima 1998;556-65.

3. Gtayson M. Deseases of the córnea. The C.V. Mosby Co. St. Louis U.S.A. 1979.

4. Paton, D, Golberg N. Managemet of ocular emergencies. W. B Saunders Company Philadelfia U.S.A. 1986

5. Pokhrel PK, Loftus SA. Ocular emergencies. Am Fam Physician 2007;76(6):829-36.
6. Simons JM. Hirertensiones Oculares, Edituorial JIMS. Barcelona, España. 1998.

7.Sampaolesi R. Glaucoma. Ed. Med. Panamericana. Buenos Aires, Argentina. 1986.

8. Pfister R. Chemical inguries of the eye. Ophthalmology 1983;9019:1243-53.

9. Hany E, EI Mekawey, et al. Epidemiology of ocular emergencies: a five year retrospective study. Clin. Ophthalmol 2011;5:955-60.

Citar como: Barriga-Salaverry G. Emergencias oftalmológicas y su manejo por el médico general. Diagnóstico(Lima).2019;58(2):72-5. DOI: https://doi.org/10.33734/diagnostico.v58i2.206

Correspondencia: Guillermo Barriga-Salaverry Correo electrónico: guillermo.barriga@upch.pe 